\title{
Real-world anticancer medications for reproductive-age women with breast cancer by using a claims database in Japan
}

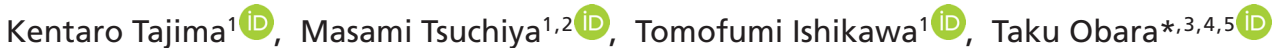 \\ \& Nariyasu Mano ${ }^{1,3}$ \\ ${ }^{1}$ Laboratory of Clinical Pharmacy, Tohoku University Graduate School of Pharmaceutical Sciences, 1-1 Seiryo-machi, Aoba-ku, \\ Sendai, Miyagi, 980-8574, Japan \\ ${ }^{2}$ Department of Pharmacy, Miyagi Cancer Center, 47-1 Nodayama, Medeshimashiote, Natori, Miyagi, 9811293, Japan \\ ${ }^{3}$ Department of Pharmaceutical Sciences, Tohoku University Hospital, 1-1, Seiryo-machi, Aoba-ku, Sendai, Miyagi, 9808574, Japan \\ ${ }^{4}$ Division of Preventive Medicine \& Epidemiology, Tohoku Medical Megabank Organization, Tohoku University, 2-1, Seiryo-machi, \\ Aoba-ku, Sendai, Miyagi, 9808573, Japan \\ ${ }^{5}$ Department of Molecular Epidemiology, Environment \& Genome Research Center, Tohoku University Graduate School of \\ Medicine, 2-1, Seiryo-machi, Aoba-ku, Sendai, Miyagi, 9808573, Japan \\ *Author for correspondence: Tel.: +81 227177 528; obara-t@hosp.tohoku.ac.jp
}

Aim: To describe real-world breast cancer medications among reproductive-age women. Patients \& methods: Using data from a Japanese claims database, anticancer prescriptions were classified into seven categories of amenorrhea risk based on fertility preservation guidelines. Results: We identified 2999 women with records of breast cancer and anticancer prescription from 2005 to 2018 . The proportions of prescriptions were as follows: high, 4.1-12.9\%; intermediate: 6.0-16.3\%; low: 0.4-2.3\%; very low/no: 0.3-12.2\%; unknown: 33.9-45.5\%; unlisted combination: 12.2-23.4\%; and unlisted drug: 12.5-26.7\%. The common drugs in the unknown category were trastuzumab $(n=1527)$, docetaxel $(n=1014)$, and paclitaxel $(n=995)$. For medications unlisted in the guidelines, various drugs and drug combinations were observed. Conclusion: Numerous anticancer drugs are currently being prescribed with insufficient evidence regarding amenorrhea risk.

Lay abstract: The ability to have children for breast cancer patients is one of the key issues of cancer survivorship, especially because recent progress in anticancer treatments has enabled patients to achieve longer survival. The fertility preservation guidelines of the American Society of Clinical Oncology (2006) introduce some anticancer treatments that carry potential risks to future fertility. In this study, the anticancer prescriptions of 2999 patients with breast cancer aged between 15 and 49 years were examined. Results showed that several medications are prescribed despite the lack of information on the risk of infertility. This suggests that further research is required to fill the evidence gap, and that decision aid through adequate counseling should be undertaken.

Tweetable abstract: A recent descriptive study by \#Japanese claims database evaluating current anticancer medications among women of reproductive age with \#breast \#cancer based on the \#ASCO \#fertility preservation guidelines.

First draft submitted: 16 October 2020; Accepted for publication: 22 January 2021; Published online: 24 February 2021

Keywords: breast cancer $\bullet$ chemotherapy $\bullet$ claims database $\bullet$ guideline $\bullet$ infertility $\bullet$ oncofertility

Breast cancer was estimated to afflict 2.4 million women globally in 2015 and was ranked as the most common cancer as well as the leading cause of cancer death [1]. Given the increase in breast cancer incidence after the age of 30 years, treatment decisions made during the reproductive period (from 15 to 49 years of age) need to be carefully considered, because important life events, such as pregnancy and delivery, occur in this time. Since recent progress in cancer treatments has enabled breast cancer patients to achieve long-term survival; and the risk of infertility after cancer treatment has become an increasingly important topic for cancer survivors [2,3].

Therapy with medications, including anticancer drugs, has been one of the essential options for treatment of both early and metastatic breast cancer [4,5]. Chemotherapy for breast cancer is currently selected based on the

Future : Medicine 
stage, expression of receptors (estrogen receptor, progesterone receptor and HER 2), and tumor biology [4,5]. It is well known that chemotherapy induces gonadal toxicity and infertility and that the risk of infertility is also related to patient age [6,7]. The mechanism by which chemotherapy causes gonadal toxicity has not been fully elucidated, yet apoptosis of primordial follicles, cortical fibrosis with damaged vasculature and depletion of primordial follicles due to recruitment of dormant follicles have been proposed to be involved [6].

Several academic societies have published guidelines or recommendations to provide information about fertility preservation [8-13]. Of these, in 2006, the American Society of Clinical Oncology (ASCO) provided general guidelines regarding the classification of risk of permanent amenorrhea in women that covered some chemotherapies [8]. While the guidelines classified alkylating drugs, including cyclophosphamide, as having the greatest risk, cyclophosphamide-containing regimens are frequently utilized in breast cancer treatments. However, to the best of our knowledge, there are no reports describing the use of each risk-classified drug aimed at women of reproductive age with breast cancer. Given recent advances in medications, it is assumed that current clinical practices may not be aligned with the medications described in the guidelines published in $2006[8]$.

The aim of this study was to describe currently prescribed medications in the context of the ASCO guidelines among women of reproductive age with breast cancer in Japanese real-world settings.

\section{Patients \& methods}

\section{Study design \& data source}

We conducted a descriptive study using a nationwide health insurance claims database provided by JMDC (Tokyo, Japan) [14]. Though this claims database does not cover the whole population of Japan, it is the largest commercially available insurance-based claims database in Japan [15] that includes the population of reproductive age. It contains inpatient, outpatient and pharmacy claims from health insurance societies in the form of structured data including demographic information, International Classification of Diseases 10th revision (ICD-10) information, surgical and medical procedure information and information on medication. Standardized disease classification and record anonymization were performed [16].

\section{Study population}

We used claims data entered between January 2005 and September 2018. Data for women of reproductive age (from 15 to 49 years [17]) who had a diagnosis of breast cancer based on the ICD-10 code C50 (malignant neoplasm of breast) were extracted. To avoid including patients with suspected breast cancer, diagnostic records with a 'primary flag' were excluded. While a primary flag is arbitrarily recorded prior to a definitive diagnosis, it is removed once the definitive diagnosis has been confirmed. For descriptive analysis, we identified cases with at least one prescription of a World Health Organization Anatomical Therapeutic Chemical (WHO-ATC) code L01 agent (antineoplastic agent) after the initial diagnosis. Considering the adolescent and young adult population [18], the subgroup aged $15-39$ years was also evaluated as a supplemental assessment.

For $a d$ hoc analysis, we also identified cases with prescriptions of anticancer drugs during the perioperative period. The perioperative population was defined as those who had L01 prescriptions between 60 and 90 days from the day of a recorded surgery. This time interval was set based on the clinically established threshold for perioperative (neoadjuvant and adjuvant) chemotherapy for early breast cancer [19-21].

\section{Classification of drugs by risk of gonadal toxicity}

Classification of gonadal toxicity risk was performed based on the guidelines from the ASCO published in 2006 (Table 1). There were seven risk categories: high, intermediate, low, very low/no, unknown, unlisted combination and unlisted drug. Since the very low/no category included only methotrexate and fluorouracil, the prescription records from this category were excluded if the drugs were combined with other drugs (e.g., cyclophosphamide, epirubicin and doxorubicin) in the high, middle or low category. In addition, if combination regimens not listed in the ASCO fertility preservation guidelines [8] but listed in the attachment of the Japanese Breast Cancer Society (JBCS) Clinical Practice Guidelines [5] were prescribed, their records were classified in the unlisted combination category.

\section{Data analysis}

The characteristics of the patients and medical institutions at diagnosis were evaluated for the study populations. Data on the age and institutional type at the time of initial breast cancer diagnosis, cohabitant status and presence of treatment records (for surgery, chemotherapy, endocrine therapy and radiation therapy) were summarized with 
Table 1. Classification of risk for permanent amenorrhea in women based on the American Society of Clinical

\section{Oncology guidelines.}

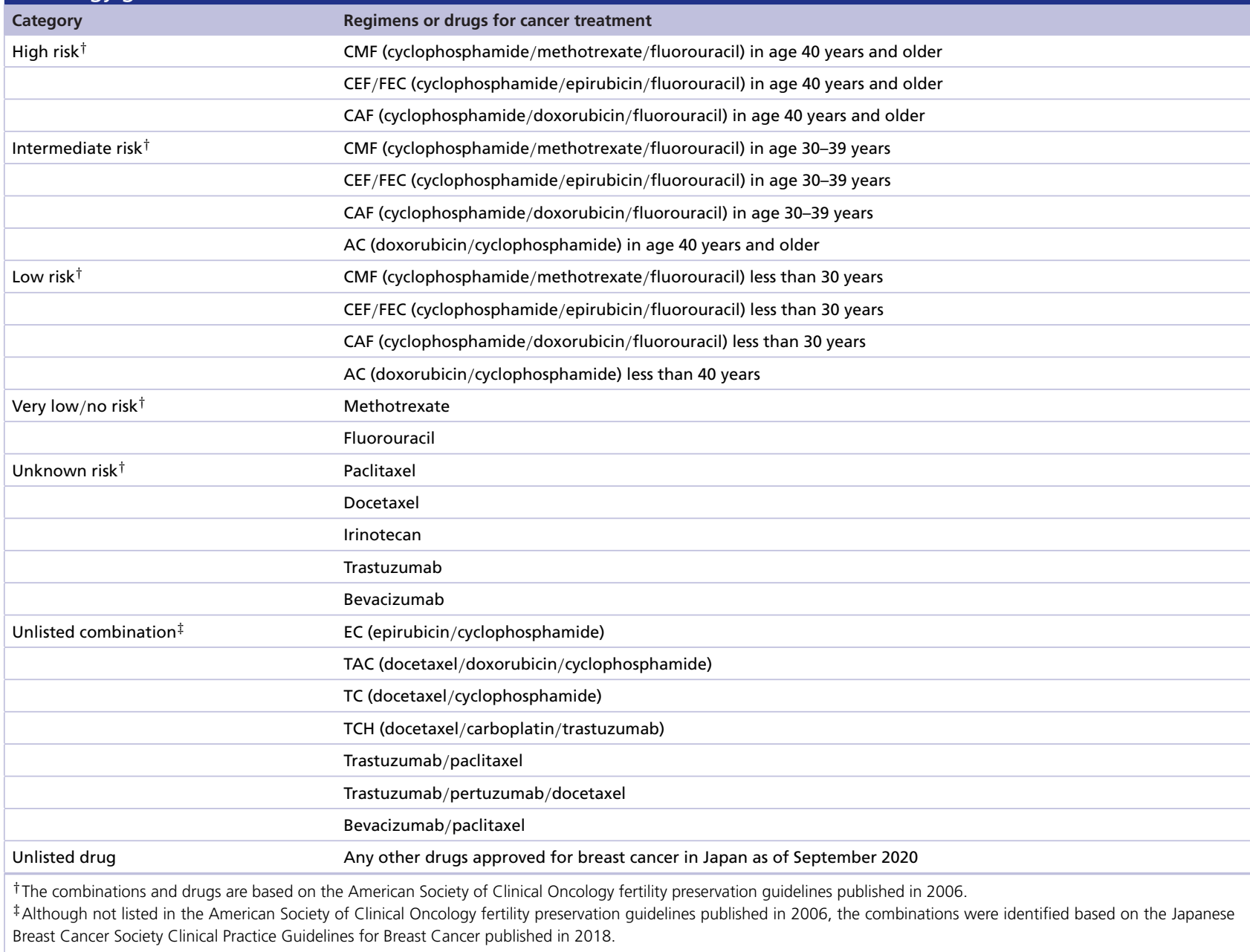

numbers and percentages (\%). Endocrine therapy was defined as a prescription with WHO-ATC code L02. The claims codes for surgery and radiation therapy related to breast cancer treatment are shown in Supplementary Table 1.

To describe the prescribed anticancer medications for women of reproductive age with breast cancer, we summarized the regimens and drugs based on their risks of gonadal toxicity by year. We defined and counted potential combination regimens as single prescriptions if each regimen-component drug was prescribed on the same date (e.g., fluorouracil/epirubicin/cyclophosphamide [CEF/FEC] was counted as one prescription despite containing three types of drugs). In this study, we did not include anticancer drugs that were not approved for breast cancer in Japan as of September 2020. Additionally, the prescribed anticancer drugs for the perioperative population were analyzed with the same method as that used for ad hoc analysis. If we could confirm only the month, rather than the exact date, for a prescription, surgery, or diagnosis in a record, we considered the date as the 15th day of the month of the claim. All analyses were performed with SAS version 9.4 (SAS Institute, Inc., NC, USA).

\section{Ethical considerations}

This study was approved by the Institutional Review Board of Tohoku University School of Medicine on 16 January 2020 (receipt number: 2019-1-686). Informed consent was not required because this was an observational study with anonymized secondary data. 
Individuals in the JMDC claims database between January 2005 and September 2018 $\mathrm{n}=5,698,893$

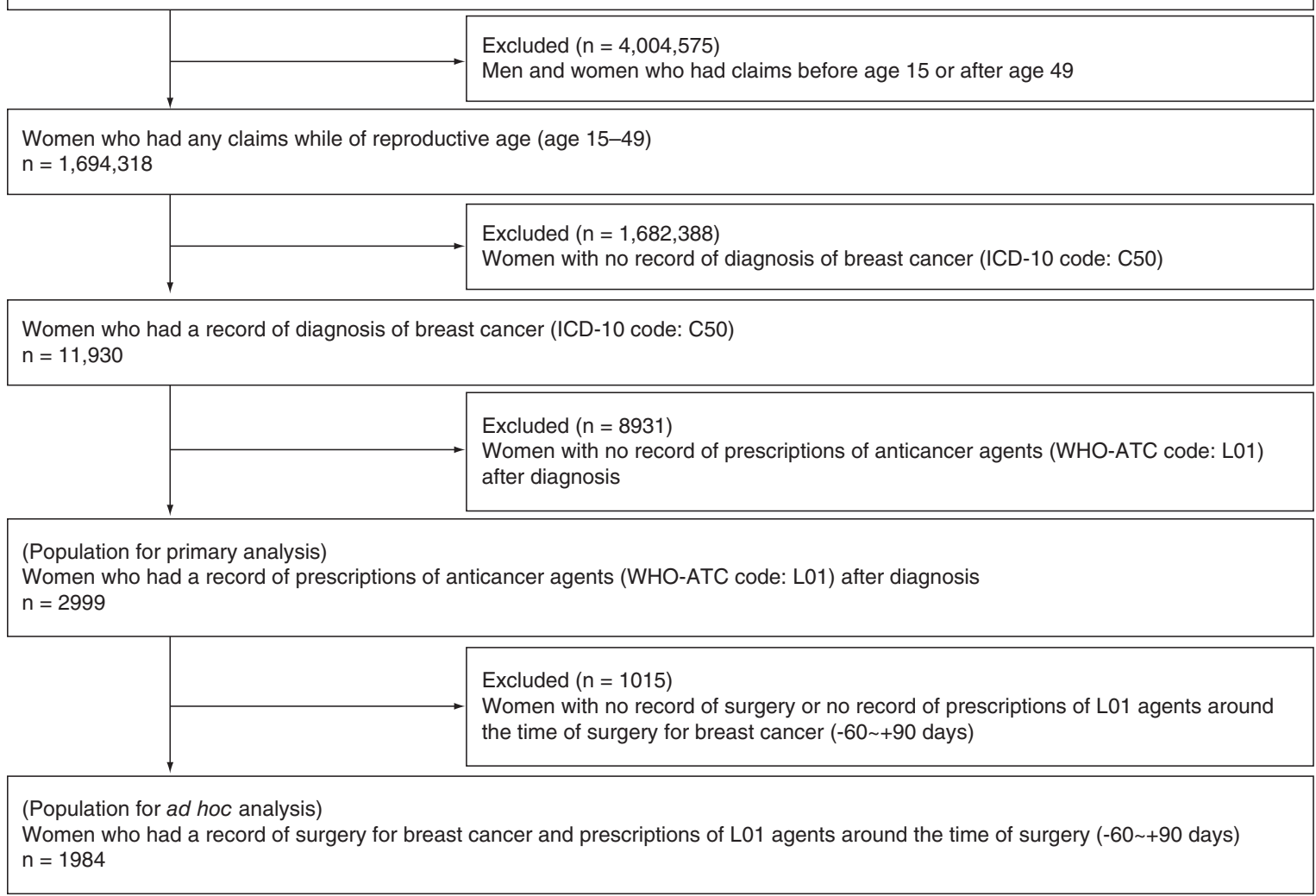

Figure 1. Flow chart of patient selection.

ICD: International Classification of Diseases; WHO-ATC: World Health Organization Anatomical Therapeutic Chemical.

\section{Results}

A total of 5,698,893 individuals were identified in the dataset between January 2005 and September 2018. Of these, 2999 women met the inclusion criteria for primary analysis, having records of prescriptions of anticancer drugs after their breast cancer diagnoses. Furthermore, 1984 women who had records of surgery for breast cancer and prescriptions of anticancer drugs around the surgery period were identified for $a d$ hoc analysis (Figure 1).

\section{Patient characteristics}

Table 2 summarizes the characteristics of patients who met the inclusion criteria for the primary analysis. At the time of diagnosis of breast cancer, most of the women were over 30 years old (23.2\% were $30-39$ years old, and $75.2 \%$ were $40-49$ years old); our dataset did not include women who were $15-19$ years old. The index year at diagnosis showed a trend of increasing diagnosis with year because the amount of data before 2009 is limited, and more than $90 \%$ of the women were diagnosed with breast cancer based on claims made after 2010 . Approximately half $(55.5 \%)$ of the initial breast cancer diagnoses were made in undesignated cancer hospitals in Japan. The percentages of women who underwent endocrine therapy, surgery and radiation therapy during the study period were $66.0,70.9$ and $48.3 \%$, respectively.

\section{Prescriptions of anticancer drugs}

Based on the classification in Table 1, the proportions of prescriptions by year are shown in Figure 2. The most frequently observed risk category each year was the unknown category, which accounted for 33.9-45.5\% of prescriptions. In addition, unlisted combination and unlisted drug categories accounted for $12.2-23.4 \%$ and $12.5-26.7 \%$ of prescriptions, respectively. On the other hand, relatively low percentages of prescriptions were in the high (4.1-12.9\%), intermediate (6.0-16.3\%), low (0.4-2.3\%) and very low/no $(0.3-12.2 \%)$ risk categories.

The detailed information on each regimen/drug classified by year is summarized in Table 3 . 


\begin{tabular}{|c|c|}
\hline & $n(\%)$ \\
\hline \multicolumn{2}{|l|}{ Age at diagnosis (years) } \\
\hline$-15-19$ & $0(0.0)$ \\
\hline$-20-29$ & $48(1.6)$ \\
\hline$-30-39$ & $697(23.2)$ \\
\hline$-40-49$ & $2254(75.2)$ \\
\hline \multicolumn{2}{|l|}{ Relationship to insured } \\
\hline - Self & $982(32.7)$ \\
\hline -Spouse & $1773(59.1)$ \\
\hline - Child & $20(0.7)$ \\
\hline - Other & $8(0.3)$ \\
\hline - Not available & $216(7.2)$ \\
\hline \multicolumn{2}{|l|}{ Index year at diagnosis } \\
\hline-2005 & $49(1.6)$ \\
\hline-2006 & $23(0.8)$ \\
\hline-2007 & $25(0.8)$ \\
\hline-2008 & $61(2.0)$ \\
\hline-2009 & $104(3.5)$ \\
\hline-2010 & $154(5.1)$ \\
\hline-2011 & $182(6.1)$ \\
\hline-2012 & $254(8.5)$ \\
\hline-2013 & $364(12.1)$ \\
\hline-2014 & $320(10.7)$ \\
\hline-2015 & $532(17.7)$ \\
\hline-2016 & $413(13.8)$ \\
\hline-2017 & 345 (11.5) \\
\hline-2018 & $173(5.8)$ \\
\hline \multicolumn{2}{|c|}{ Status of designated cancer hospital at diagnosis } \\
\hline - Prefectural hospital & $281(9.4)$ \\
\hline - Regional hospital & $1055(35.2)$ \\
\hline - Undesignated hospital & $1663(55.5)$ \\
\hline \multicolumn{2}{|l|}{ Other cancer treatments } \\
\hline - Hormonal therapy & $1980(66.0)$ \\
\hline - Surgery & $2126(70.9)$ \\
\hline - Radiation therapy & $1448(48.3)$ \\
\hline
\end{tabular}

CEF/FEC and doxorubicin/cyclophosphamide (AC) were mainly observed in the high, intermediate and low categories, while other regimens (cyclophosphamide/methotrexate/fluorouracil [CMF] and cyclophosphamide/doxorubicin/fluorouracil [CAF]) were relatively rare. The use of methotrexate and fluorouracil (not prescribed in cyclophosphamide-, epirubicin- or doxorubicin-containing regimens, including CMF, CEF/FEC and CAF), in the very low/no category, was also very limited. Within the unknown category, trastuzumab $(\mathrm{n}=1527)$, docetaxel $(\mathrm{n}=1014)$ and paclitaxel $(\mathrm{n}=995)$ were frequently observed. In the unlisted combination category, which featured regimens not listed in the ASCO fertility preservation guidelines, epirubicin/cyclophosphamide (EC, $\mathrm{n}=609$ ), docetaxel/doxorubicin/cyclophosphamide (TAC, $\mathrm{n}=6$ ), docetaxel/cyclophosphamide $($ TC, $\mathrm{n}=573$ ), docetaxel/carboplatin/trastuzumab $(\mathrm{TCH}, \mathrm{n}=16)$, trastuzumab + paclitaxel $(\mathrm{n}=285)$, trastuzumab + pertuzumab + docetaxel $(\mathrm{n}=98)$ and bevacizumab + paclitaxel $(\mathrm{n}=294)$ were observed. Moreover, various kinds of anticancer drugs were identified in the unlisted drug category. Capecitabine $(\mathrm{n}=477)$, tegafur combinations $(\mathrm{n}=409)$, eribulin $(\mathrm{n}=275)$, vinorelbine $(\mathrm{n}=173)$, pertuzumab $(\mathrm{n}=149)$, gemcitabine $(\mathrm{n}=133)$, lapatinib $(\mathrm{n}=106)$ and trastuzumab emtansine $(\mathrm{n}=100)$ had more than 100 prescriptions in the dataset. Regarding the 15-39 years age subgroup (Supplementary Figure 1 \& Supplementary Table 2), the same trend was observed. 


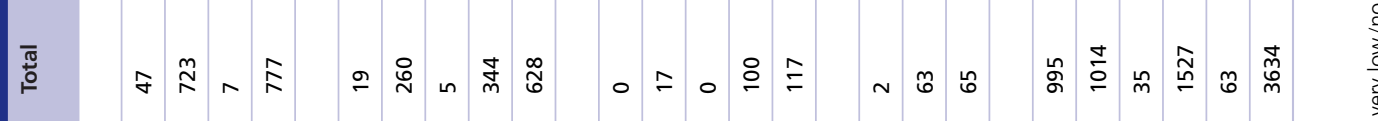

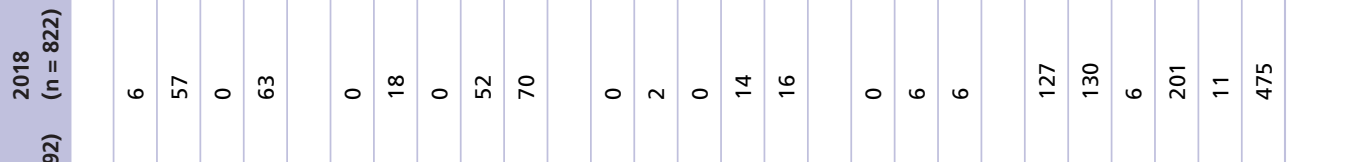

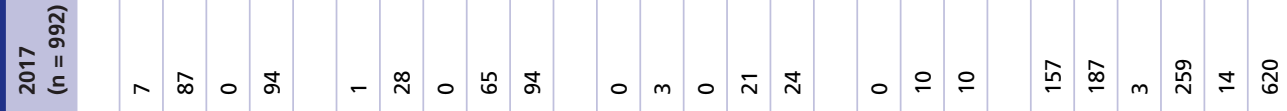

๑ั

ํㅜㄹ

ฉ

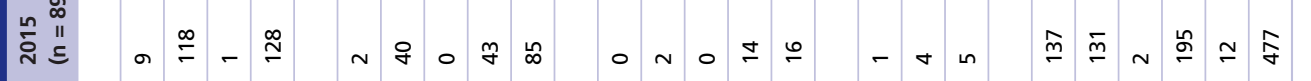

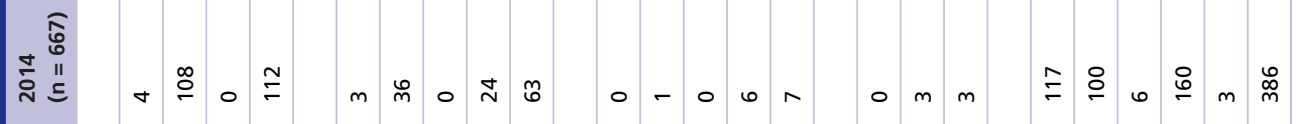

更

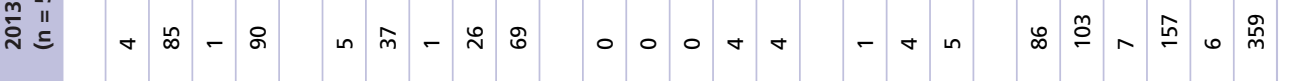

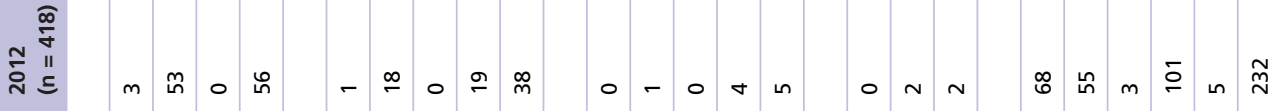

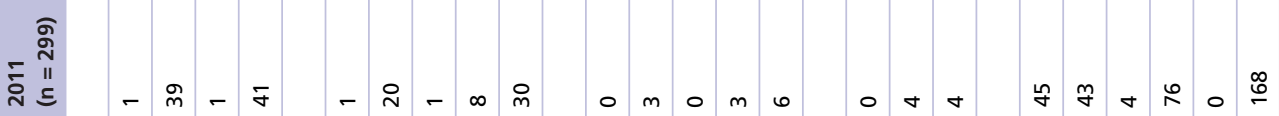

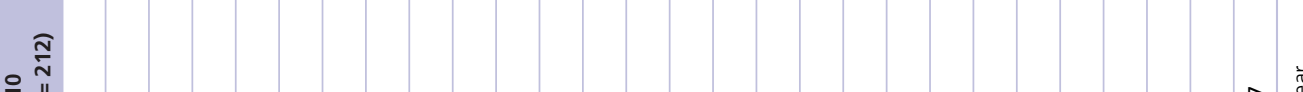

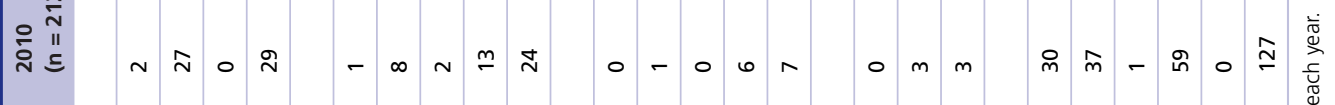

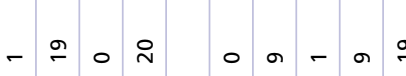

- $00 \mathrm{~m} m$ o n

ละ $2-70 \%$

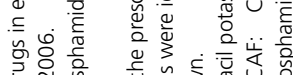

‡̊

t
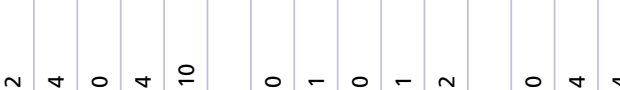

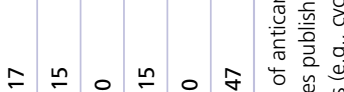

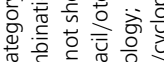

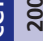

- n

政

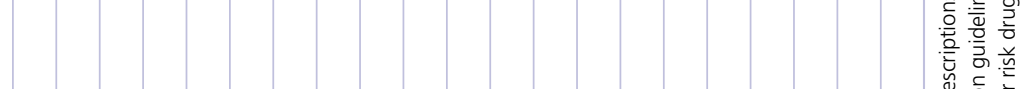

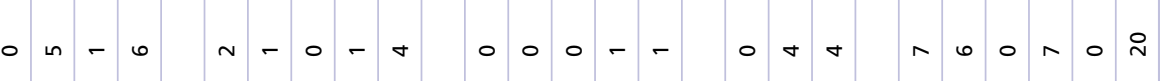

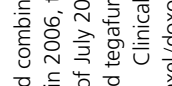




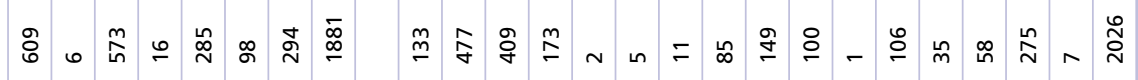

离

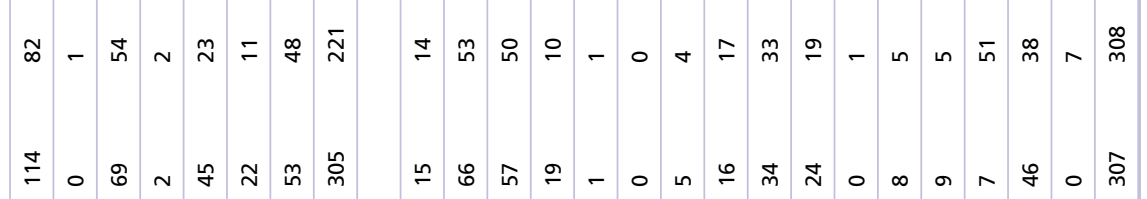

สू๊

突

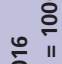

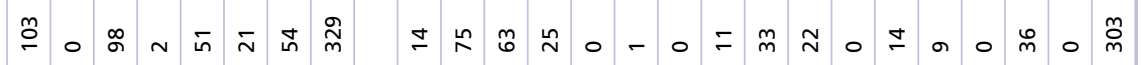

要

点

$\infty$ ๙

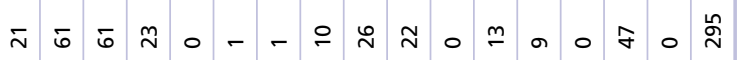

:

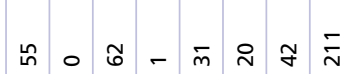

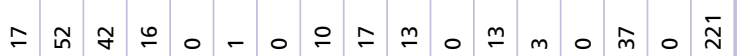

品

L̊ $0 \tilde{\varphi}+\bar{m} m \stackrel{\infty}{\stackrel{\infty}{\infty}}$

I

ำ

กับ

ริ

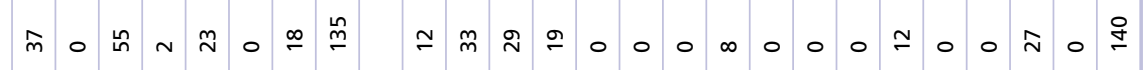

¿ัं

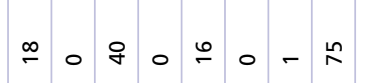

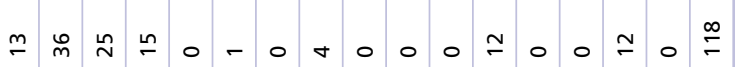

$\simeq 0 \simeq-n 00 \%$

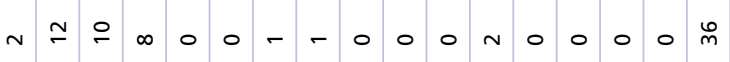

$m 0 m-000 \hat{N}$
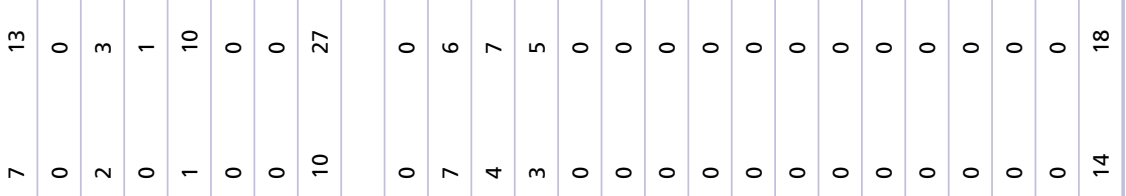

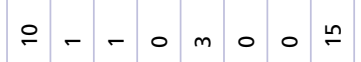

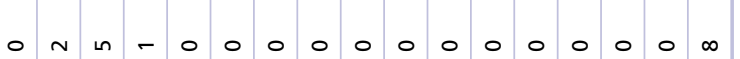

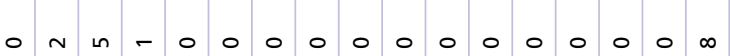

$m-00 n$
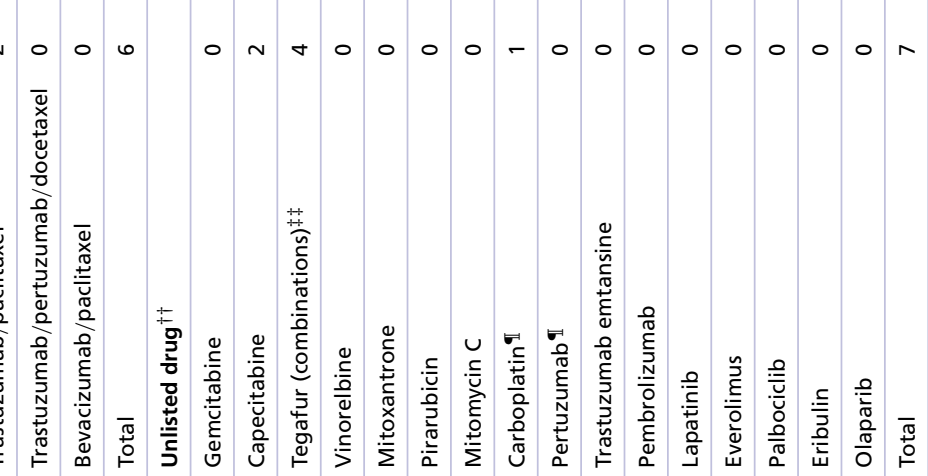


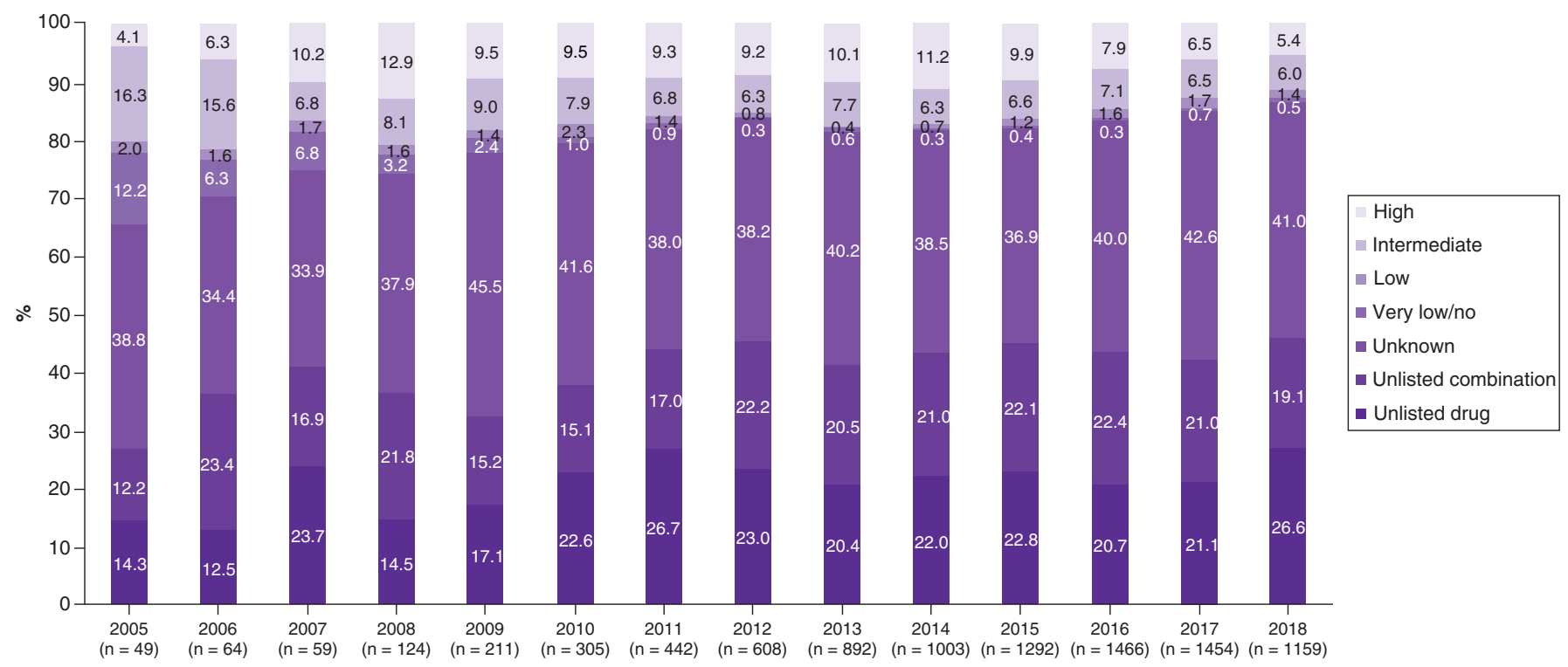

Figure 2. Proportions of anticancer drugs by year. The numbers on the bars represent the percentages of prescriptions in each year. Duplicates such as the same regimens/drugs used in the same patient in the same year were removed.

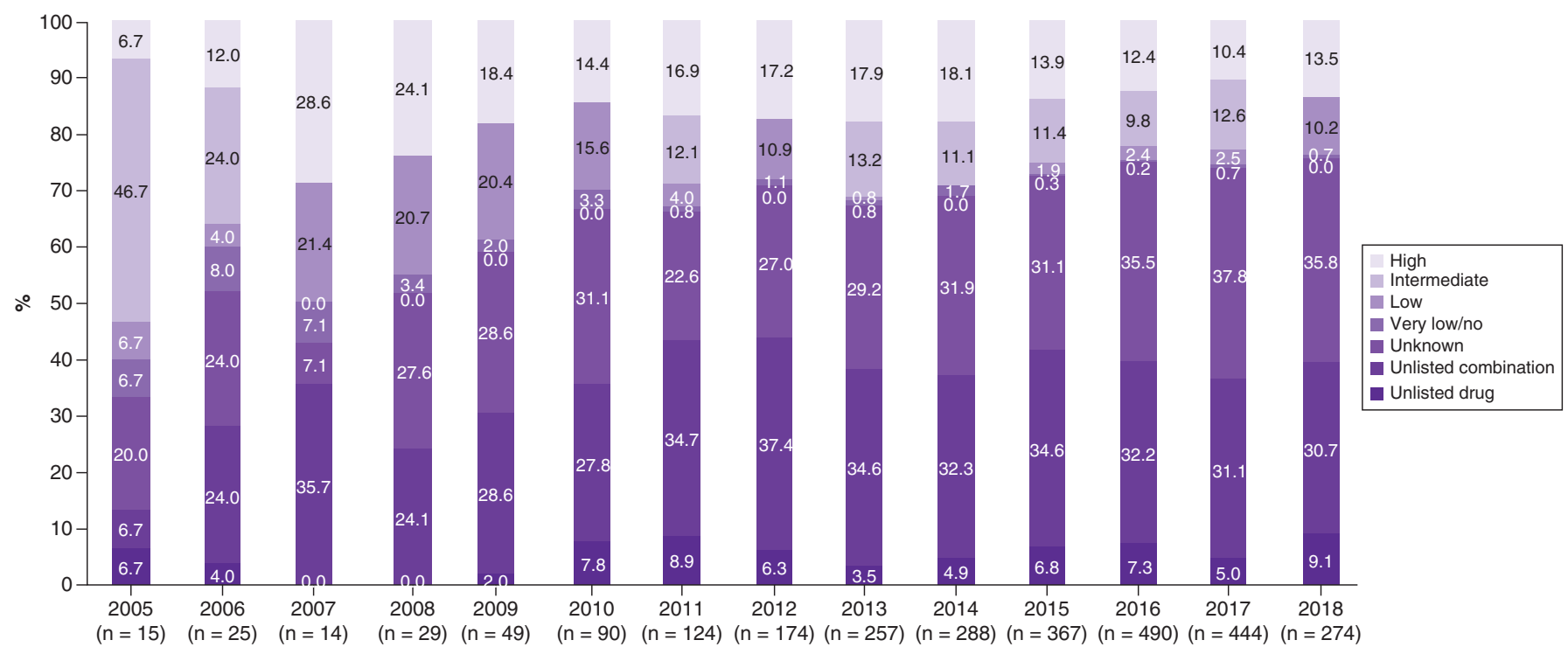

Figure 3. Proportions of anticancer drugs by year (ad hoc analysis for the perioperative population). The numbers on the bars represent the percentages of prescriptions in each year. Duplicates such as the same regimens/drugs in the same patient in the same year were removed.

However, there were no prescriptions in the high-risk category because the category was only relevant to women aged 40 and above.

The proportions of prescriptions in the high, intermediate and unlisted combination categories were generally higher in the ad hoc analysis than in the primary analysis, while those in the unknown and unlisted drug categories were lower (Figure 3). The prescriptions of detailed regimens and drugs for breast cancer seemed to have similar tendencies in the ad hoc and primary analyses (Table 4). Furthermore, the proportions of the perioperative prescriptions in the neoadjuvant setting and the adjuvant setting were 22.7 and $77.3 \%$, respectively. 


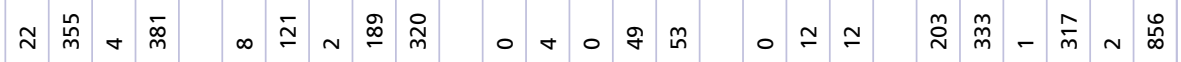

శึ

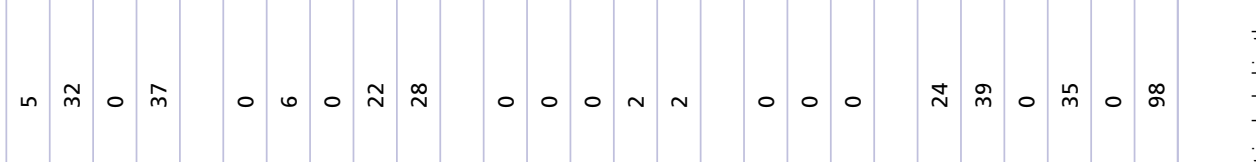

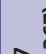

㝘

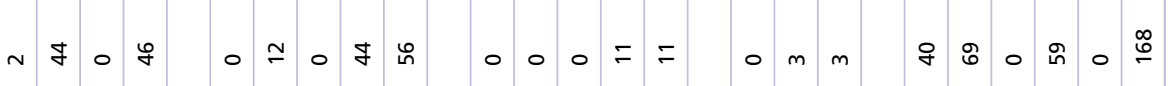

음

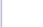

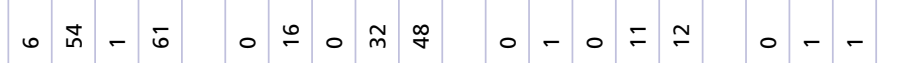

+ ร 0

- $-0 \approx$ ก
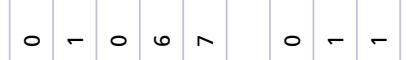

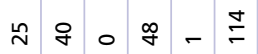

-

$-\stackrel{\varphi}{-} \stackrel{\varphi}{m}$

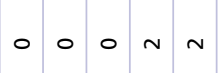

0 N N

$\simeq \stackrel{m}{m}$ o $\stackrel{\infty}{N}$

- ำ 0
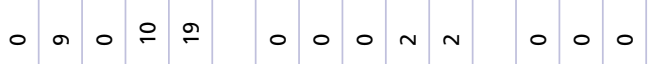

$J=0 \div 0$ F

$0 \bar{N} 0 \bar{N} \quad 0 \quad m \quad 0 \quad n \stackrel{m}{n}$

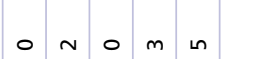

o - n $=0 \simeq 0 \stackrel{\infty}{N}$
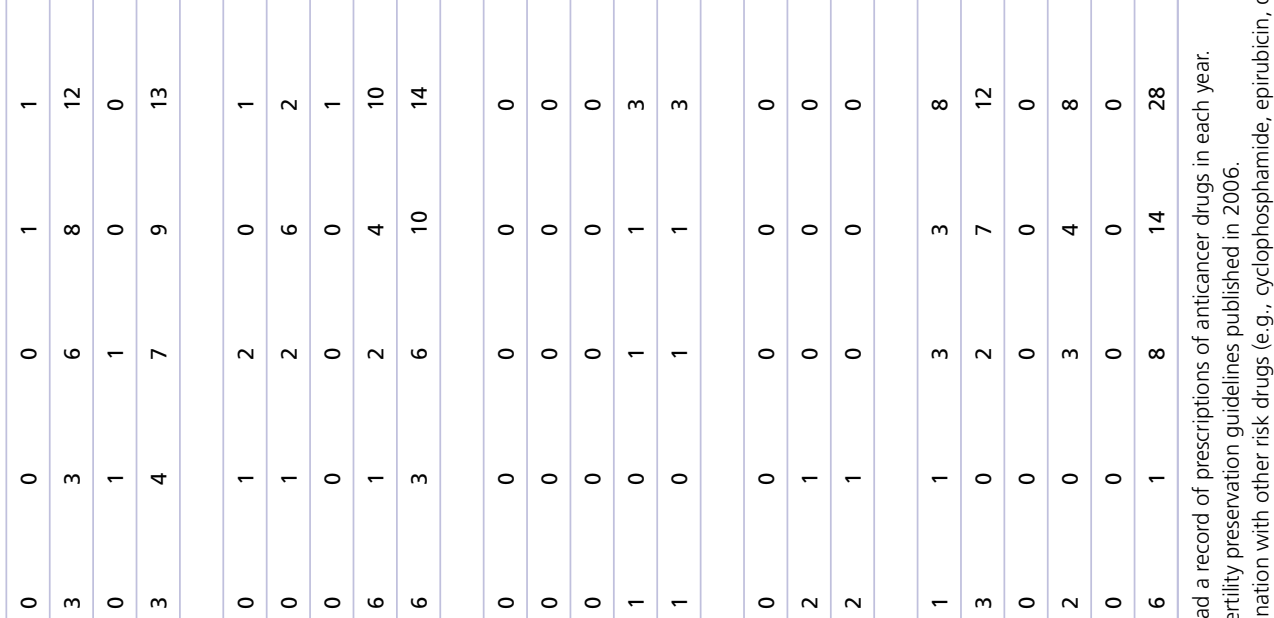

$\circ \mathrm{m} \circ \mathrm{m}$
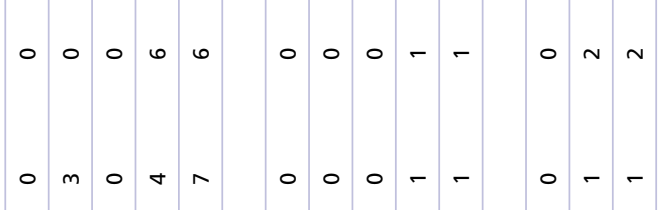

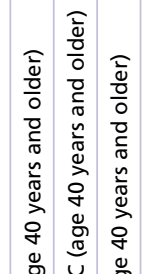

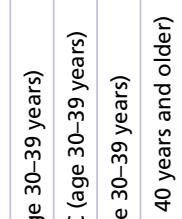

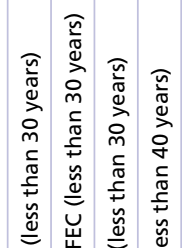




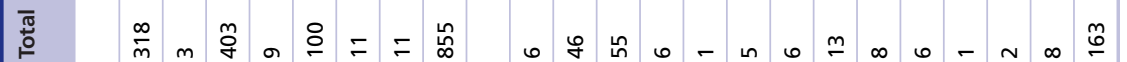
$\stackrel{\text { ป }}{\text { ป }}$

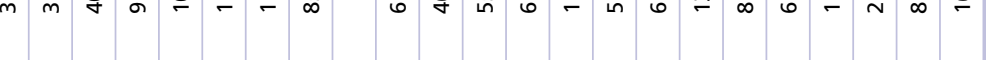




\section{Discussion}

To the best of our knowledge, this is the first evaluation of chemotherapy trends in a real-world setting among reproductive-age women with breast cancer in the context of the fertility preservation guidelines published by the ASCO in 2006. The value of this study is in its clarification of how current clinical prescriptions are classified into risk categories and in its identification of the anticancer drugs that are not listed in the guidelines.

Consistent with existing literature [22,23], in this study, we revealed that the CMF and CAF regimens, which are mentioned in the ASCO fertility preservation guidelines, are not frequently used in current treatment settings. On the other hand, CEF/FEC and AC regimens were commonly prescribed. In the unknown risk category according to the guidelines, taxanes (paclitaxel and docetaxel) and trastuzumab were frequently prescribed. These results support the idea that taxane-based regimens and trastuzumab-based regimens are essential in breast cancer treatment $[4,5]$. The available clinical evidence demonstrates the efficacy of taxanes plus cyclophosphamide with or without anthracyclines and indicates that trastuzumab should be added for HER2-positive cancer [22,24-26]. More importantly, we also found that many combination regimens (e.g., EC, TC) and drugs (e.g., capecitabine, tegafur combinations, pertuzumab) that are not described in the ASCO fertility preservation guidelines are prescribed to women of reproductive age in real-world clinical practice (Table 3). These observations suggest that the guidelines are expected to cover the current medications, such as the anticancer drugs and combination regimens shown above. In general, although the number of prescriptions in the dataset increased year over year due to enlargement of the JMDC claims database, the tendency did not change each year; thus, it appears that the year of the prescription did not impact the results above.

Because perioperative chemotherapy may reflect treatment for early breast cancer, we also evaluated the prescriptions given around the time of surgery through ad hoc analysis. Fertility preservation may be actively considered for patients with early breast cancer, although patients with metastatic breast cancer should also be provided with the opportunity to discuss fertility preservation [27]. We observed fewer prescriptions in the ad hoc analysis of bevacizumab and anticancer drugs, which were classified in the unlisted drug category, compared with the primary analysis (Table 4). The results suggested that most perioperative prescriptions in the ad hoc analysis were used for early breast cancer. Thus, prescriptions including bevacizumab and many unlisted drugs that are only approved for metastatic or recurrent breast cancer might be less common than other drugs. Nevertheless, numerous anticancer drugs were classified in the unknown and unlisted combination categories. It is assumed that both healthcare providers and patients must often communicate and make treatment decisions without adequate information on the risk of infertility associated with such anticancer drugs, regardless of the stage of breast cancer.

The ASCO fertility preservation guidelines have been updated twice to improve survivorship for patients with cancer $[11,12]$. However, since the guidelines were first published in 2006 , the classification of the gonadal toxicity risk of chemotherapy has not been updated. To date, large studies evaluating infertility caused by anticancer drugs have been extremely limited, but a few have been conducted on early breast cancer [23,28-30]. With regard to taxane-containing regimens, one study showed that the incidence rate of amenorrhea among patients on the TAC regimen was significantly higher than that among patients on the CAF regimen (61.7 vs $52.4 \%$; $=0.007)$ [23]; however, another study did not show an increase in amenorrhea risk due to addition of paclitaxel or trastuzumab [28]. Moreover, a further study showed the incidence rates of amenorrhea among patients treated with AC followed by docetaxel, TAC and doxorubicin plus docetaxel were 69.8, 57.7 and 37.9\%, respectively. Therefore, it seems that longer treatment durations such as those necessary for AC followed by docetaxel are somewhat associated with amenorrhea in patients undergoing taxane-containing regimens [29]. A recent report has also highlighted that the amenorrhea risks of trastuzumab and lapatinib do not differ [31]. Overall, although more clinical evidence is needed, incorporation of this newly available evidence into the ASCO fertility preservation guidelines might be helpful especially for healthcare providers operating outside the field of oncofertility.

Since most medications for breast cancer are used in combination regimens, it is difficult to determine which specific drug contributes to infertility. However, as shown in our study, various anticancer drugs have been used for which there is no evidence of contributions to infertility. In addition, various new targeted therapies in the neoadjuvant setting and the adjuvant setting have now been developed and are expected to be approved for early breast cancer [32]. Continuous efforts to update existing evidence are needed. Regarding these various anticancer drugs, it is expected that new evidence on both amenorrhea and ovarian reserve testing, such as serum anti-Müllerian hormone levels, will be accumulated for an adequate assessment of infertility [33]. 
Individualized counseling based on the benefits and harms of anticancer drugs, including the impact on fertility, pre-treatment ovarian reserve, the desire for future childbearing, is a critical element of clinical practice. Nonetheless, in both Japan and Western countries, well-informed communication between healthcare providers and patients regarding fertility preservation before cancer treatment has been acknowledged to be insufficient [34-37]. To facilitate the communication and improve the decision-making process, new evidence regarding the risk of infertility should be utilized.

We are aware that this study has several limitations. First, the accuracy of the diagnoses of breast cancer in the claims data is uncertain, and some of the diagnoses might be missing as claims data are not collected for research purposes. However, we included only diagnoses with prescriptions of anticancer drugs and excluded diagnoses with primary flags. Therefore, we believe that patient successful selection was achieved. Second, the claims database does not include some treatment-related information. The information regarding fertility care and diagnosis of infertility is not contained in the claims database because fertility treatment is not currently covered by insurance in Japan. In addition, since detailed information on characteristics such as the stage, subtype, or biology of breast cancer is not included in the claims, it was difficult to consider these factors, which could affect treatment decisions. This limitation implies that the ad hoc analysis did not necessarily reflect the treatment of early breast cancer, although surgery for metastatic breast cancer was limited. Third, the insurance-based claims database is not designed to follow up patients who withdraw from the health insurance. Despite this, the database has a great advantage over hospital-based databases: insured patients and their dependents can be followed even when they are transferred to another hospital unless they withdraw from the insurance society. Finally, with regard to the combination regimens, we evaluated the combinations listed in the ASCO fertility preservation guidelines and the JBCS Clinical Practice Guidelines. Thus, although standard regimens were covered, the combination regimens that were not included in these guidelines were not evaluated in this study. Further research using different databases and investigations in clinical settings should be performed to provide more detailed information, as well as information on obstetric outcomes including pregnancy and delivery.

\section{Conclusion}

Our observations reveal the prescription of various combination regimens and drugs that were not listed in the 2006 ASCO fertility preservation guidelines for women of reproductive age with breast cancer in a real-world setting in Japan. It is assumed that various medications are prescribed despite a lack of information on the risk of infertility. We believe that to enhance effective counseling and decision-making support, information on the risk of infertility should be continuously updated following current clinical practices. Moreover, further research is required to generate more detailed clinical evidence that can be used to evaluate the risk of infertility associated with cancer medications.

Supplementary data

To view the supplementary data that accompany this paper please visit the journal website at: www.futuremedicine.com/doi/suppl/10.2217/fon-2020-1053

\section{Author contributions}

All authors have contributed to this work and approved the final manuscript. K Tajima was responsible for study conception. K Tajima, M Tsuchiya and T Obara designed this study and performed the data analysis. $K$ Tajima wrote the first draft of the manuscript, and M Tsuchiya, T Ishikawa, T Obara and N Mano contributed to interpretation of data and critically revised the manuscript.

Financial \& competing interests disclosure

This study was supported in part by a Grant-in-Aid from the Kurokawa Cancer Research Foundation. K Tajima is an employee of Pfizer Japan, Inc. T Ishikawa is an employee of Pfizer R\&D Japan. The authors have no other relevant affiliations or financial involvement with any organization or entity with a financial interest in or financial conflict with the subject matter or materials discussed in the manuscript apart from those disclosed.

No writing assistance was utilized in the production of this manuscript. 


\section{Ethical conduct of research}

This study was approved by the Institutional Review Board of the Tohoku University School of Medicine on 16 January 2020 (receipt number: 2019-1-686). Informed consent was not required because this was an observational study with anonymized secondary data.

\section{Open access}

This work is licensed under the Attribution-NonCommercial-NoDerivatives 4.0 Unported License. To view a copy of this license, visit http://creativecommons.org/licenses/by-nc-nd/4.0/

\section{Summary points}

- The American Society of Clinical Oncology (ASCO) published fertility preservation guidelines specifying amenorrhea risk categories for medications including chemotherapy in 2006.

- This study aimed to describe currently prescribed medications in the context of the ASCO guidelines among women of reproductive age with breast cancer by using a nationwide claims database in Japan between January 2005 and September 2018.

- Based on the ASCO guidelines, the anticancer prescriptions for each year were classified into seven categories of amenorrhea risk: high, intermediate, low, very low/no, unknown, unlisted combination and unlisted drug.

- In the dataset, 2999 women met the inclusion criteria for primary analysis.

- The proportions of prescriptions in the different categories were as follows: high; $4.1-12.9 \%$; intermediate; 6.0-16.3\%; low; 0.4-2.3\%; very low/no; 0.3-12.2\%; unknown; 33.9-45.5\%; unlisted combination; 12.2-23.4\%; and unlisted drug; $12.5-26.7 \%$.

- The common drugs in the unknown category were trastuzumab $(n=1527)$, docetaxel $(n=1014)$ and paclitaxel $(n=995)$.

- For medications not described in the ASCO guidelines, various drugs and drug combinations were observed, including epirubicin/cyclophosphamide $(E C, n=609)$, docetaxel/cyclophosphamide $(T C, n=573)$, capecitabine $(n=477)$ and tegafur combinations $(n=409)$.

- Various anticancer drugs for which there is insufficient evidence about amenorrhea risk are currently prescribed to reproductive-age women with breast cancer.

- To support effective counseling and decision making regarding fertility preservation, information on the risk of infertility should be updated based on the current clinical practice, and further clinical evidence about drug-induced infertility should be obtained.

\section{References}

Papers of special note have been highlighted as: $\bullet$ of interest; $\bullet \bullet$ of considerable interest

1. Collaboration GBODC. Global, Regional, and national cancer incidence, mortality, years of life lost, years lived with disability, and disability-adjusted life-years for 32 cancer groups, 1990 to 2015: a systematic analysis for the global burden of disease study. JAMA Oncol. 3(4), 524-548 (2017).

2. Benedict C, Shuk E, Ford JS. Fertility issues in adolescent and young adult cancer survivors. J. Adolesc. Young Adult Oncol. 5(1), 48-57 (2016).

3. Sobota A, Ozakinci G. Fertility and parenthood issues in young female cancer patients - a systematic review. J. Cancer Surviv. 8(4), 707-721 (2014).

4. National Comprehensive Cancer Network. NCCN Clinical Practice Guidelines in Oncology. Breast Cancer Version 4 (2020). https://www.nccn.org/professionals/physician_gls/default.aspx

5. The Japanese Breast Cancer Society. Clinical Practice Guidelines for Breast Cancer 2018 (in Japanese). Kanehara \& Co., LTD, Tokyo, Japan (2018).

- This is the latest clinical practice guidelines for breast cancer in Japan.

6. Meirow D, Biederman H, Anderson RA, Wallace WH. Toxicity of chemotherapy and radiation on female reproduction. Clin. Obstet. Gynecol. 53(4), 727-739 (2010).

7. Ben-Aharon I, Shalgi R. What lies behind chemotherapy-induced ovarian toxicity? Reproduction 144(2), 153-163 (2012).

- This is a useful summary of assumed mechanism of various chemotherapy-induced infertility.

8. Lee SJ, Schover LR, Partridge AH et al. American Society of Clinical Oncology recommendations on fertility preservation in cancer patients. J. Clin. Oncol. 24(18), 2917-2931 (2006).

-. This is the world's first guidelines on fertility preservation in patients with cancer, and the only general guidelines regarding the classification of risk of permanent amenorrhea in women that covered some chemotherapies. 
9. Woodruff TK. The Oncofertility Consortium - addressing fertility in young people with cancer. Nat. Rev. Clin. Oncol. 7(8), 466-475 (2010).

10. Klemp JR, Kim SS. Fertility preservation in young women with breast cancer. J. Assist. Reprod. Genet. 29(6), 469-472 (2012).

11. Loren AW, Mangu PB, Beck LN et al. Fertility preservation for patients with cancer: American Society of Clinical Oncology clinical practice guideline update. J. Clin. Oncol. 31(19), 2500-2510 (2013).

12. Oktay K, Harvey BE, Partridge AH et al. Fertility preservation in patients with cancer: ASCO Clinical Practice Guideline update. J. Clin. Oncol. 36(19), 1994-2001 (2018).

- This is the latest American Society of Clinical Oncology (ASCO) fertility preservation guideline.

13. Suzuki N. Clinical practice guidelines for fertility preservation in pediatric, adolescent, and young adults with cancer. Int. J. Clin. Oncol. 24(1), 20-27 (2019).

-• This review paper gives an overview of clinical practice guidelines for fertility preservation in pediatric, adolescent and young adults with cancer including Japan.

14. JMDC Inc. https://www.jmdc.co.jp/en/index

15. Pharmacoepidemiology \& Database Taskforce, Japanese Society for Pharmacoepidemiology. Survey of Japanese databases in Japan available for clinical/pharmacoepidemiology (2020). http://www.jspe.jp/committee/020/0210/

16. Kimura S, Sato T, Ikeda S, Noda M, Nakayama T. Development of a database of health insurance claims: standardization of disease classifications and anonymous record linkage. J. Epidemiol. 20(5), 413-419 (2010).

17. World Health Organization. Infertility definitions and terminology. https://www.who.int/teams/sexual-and-reproductive-health-and-res earch/areas-of-work/fertility-care/inf ertility-definitions-and-terminology

18. National Comprehensive Cancer Network. NCCN Clinical Practice Guidelines in Oncology. Adolescent and Young Adult (AYA) Oncology version 1. (2021). https://www.nccn.org/professionals/physician_gls/pdf/aya.pdf

19. Yu KD, Huang S, Zhang JX, Liu GY, Shao ZM. Association between delayed initiation of adjuvant CMF or anthracycline-based chemotherapy and survival in breast cancer: a systematic review and meta-analysis. BMC Cancer 13, 240 (2013).

20. Raphael MJ, Biagi JJ, Kong W, Mates M, Booth CM, Mackillop WJ. The relationship between time to initiation of adjuvant chemotherapy and survival in breast cancer: a systematic review and meta-analysis. Breast Cancer Res. Treat. 160(1), 17-28 (2016).

21. Gagliato DM, Lei X, Giordano SH et al. Impact of delayed neoadjuvant systemic chemotherapy on overall survival among breast cancer patients. Oncologist 25(9), 749-757 (2020).

22. Peto R, Davies C, Godwin J et al. Comparisons between different polychemotherapy regimens for early breast cancer: meta-analyses of long-term outcome among 100,000 women in 123 randomised trials. Lancet 379(9814), 432-444 (2012).

23. Martin M, Pienkowski T, Mackey J et al. Adjuvant docetaxel for node-positive breast cancer. N. Engl. J. Med. 352(22), 2302-2313 (2005).

24. Jones S, Holmes FA, O'Shaughnessy J et al. Docetaxel with cyclophosphamide is associated with an overall survival benefit compared with doxorubicin and cyclophosphamide: 7-year follow-up of US oncology research trial 9735. J. Clin. Oncol. 27(8), 1177-1183 (2009).

25. De Laurentiis M, Cancello G, D’Agostino D et al. Taxane-based combinations as adjuvant chemotherapy of early breast cancer: a meta-analysis of randomized trials. J. Clin. Oncol. 26(1), 44-53 (2008).

26. Moja L, Tagliabue L, Balduzzi S et al. Trastuzumab containing regimens for early breast cancer. Cochrane Database Syst. Rev. 2012(4), Cd006243 (2012).

27. Debono DJ, Kohnke JM, Helft PR. Addressing fertility in patients with advanced cancer: how the quality oncology practice initiative standards and ASCO guidelines facilitate ethical communication. J. Oncol. Pract. 5(6), 298-300 (2009).

28. Abusief ME, Missmer SA, Ginsburg ES, Weeks JC, Partridge AH. The effects of paclitaxel, dose density, and trastuzumab on treatment-related amenorrhea in premenopausal women with breast cancer. Cancer 116(4), 791-798 (2010).

29. Ganz PA, Land SR, Geyer CE Jr et al. Menstrual history and quality-of-life outcomes in women with node-positive breast cancer treated with adjuvant therapy on the NSABP B-30 trial. J. Clin. Oncol. 29(9), 1110-1116 (2011).

- This is one of the key clinical trials of adjuvant therapy for breast cancer as well as suggesting longer treatment durations are somewhat associated with amenorrhea among taxane-containing regimens.

30. Swain SM, Land SR, Ritter MW et al. Amenorrhea in premenopausal women on the doxorubicin-and-cyclophosphamide-followed-by-docetaxel arm of NSABP B-30 trial. Breast Cancer Res. Treat. 113(2), 315-320 (2009).

31. Lambertini M, Campbell C, Bines J et al. Adjuvant anti-HER2 therapy, treatment-related amenorrhea, and survival in premenopausal HER2-positive early breast cancer patients. J. Natl Cancer Inst. 111(1), 86-94 (2019).

32. Pondé F, Zardavas D, Piccart M. Progress in adjuvant systemic therapy for breast cancer. Nat. Rev. Clin. Oncol. 16(1), 27-44 (2019).

33. Tal R, Seifer DB. Ovarian reserve testing: a user's guide. Am. J. Obstet. Gynecol. 217(2), 129-140 (2017).

34. Shimizu C, Bando H, Kato T, Mizota Y, Yamamoto S, Fujiwara Y. Physicians' knowledge, attitude, and behavior regarding fertility issues for young breast cancer patients: a national survey for breast care specialists. Breast Cancer 20(3), 230-240 (2013). 
35. Furui $\mathrm{T}$, Takai $\mathrm{Y}$, Kimura $\mathrm{F}$ et al. Fertility preservation in adolescent and young adult cancer patients: from a part of a national survey on oncofertility in Japan. Reprod. Med. Biol. 18(1), 97-104 (2019).

-. This national survey in Japan highlights the importance of information provided on risk of drug-induced infertility before cancer treatment to enhance patient decision making.

36. Duffy CM, Allen SM, Clark MA. Discussions regarding reproductive health for young women with breast cancer undergoing chemotherapy. J. Clin. Oncol. 23(4), 766-773 (2005).

37. Niemasik EE, Letourneau J, Dohan D et al. Patient perceptions of reproductive health counseling at the time of cancer diagnosis: a qualitative study of female California cancer survivors. J. Cancer Surviv. 6(3), 324-332 (2012). 